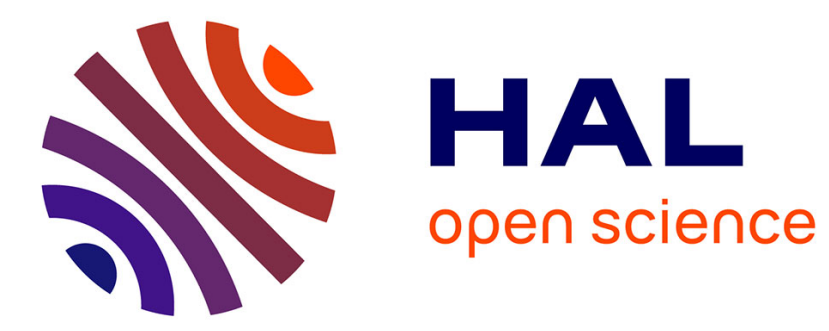

\title{
Nouveautés fiscales
}

Raymond Viney

\section{To cite this version:}

Raymond Viney. Nouveautés fiscales. Revue forestière française, 1960, 3, pp.212-215. $10.4267 / 2042 / 24265$. hal-03389691

\section{HAL Id: hal-03389691 https://hal.science/hal-03389691}

Submitted on 21 Oct 2021

HAL is a multi-disciplinary open access archive for the deposit and dissemination of scientific research documents, whether they are published or not. The documents may come from teaching and research institutions in France or abroad, or from public or private research centers.
L'archive ouverte pluridisciplinaire HAL, est destinée au dépôt et à la diffusion de documents scientifiques de niveau recherche, publiés ou non, émanant des établissements d'enseignement et de recherche français ou étrangers, des laboratoires publics ou privés. 


\section{NOUVEAUTÉS FISCALES}

PAR

R. VINEY

La Loi de Finances pour 1960, parue au Journal Officiel du 29 décembre 1959, et la loi du 28 décembre 1959 portant réforme du contentieux fiscal ont profondément modifié le régime antérieur des impôts et des droits de succession.

Nous ne saurions trop nous réjouir de certaines dispositions qui ont été prises en faveur des propriétaires forestiers.

Malheureusement l'esprit de simplification n'est pas la caractéristique des officines où se préparent les textes financiers. Il est donc très difficile de voir les répercussions possibles de toutes les innovations édictées et quelle sera la charge fiscale qui pèsera dorénavant sur le mètre cube de bois produit.

Nous résumerons brièvement les réformes:

1) Suppression de la taxe proportionnelle et de la surtaxe progressive et remplacement par un impôt unique sur le revenu des personnes physiques.

2) Modification des barêmes et des taux.

3) Suppression de l'autonomie des différentes catégories de revenu.

4) Etablissement d'un régime spécial pour les revenus de 1959.

5) Création d'une taxe complémentaire temporaire sur certains revenus qui doit prendre fin au $1^{\text {er }}$ janvier 1962.

6) Allègement des taux de droits de succession et donation pour les successions en ligne directe (époux, ascendants et ayants).

7) Aggravation du taux pour les successions en ligne collatérale (frères, sceurs, oncles, tantes, cousins).

8) Suppression de la taxe spéciale sur les successions et donations dite " Ramadier ) créée par la loi du 30 juin 1956.

9) Extension aux successions de la loi "Sérot » accordant la réduction des $3 / 4$ des droits de mutation.

10) Majoration de la plupart des droits fixes de timbre et d'enregistement.

11) Modification cle certaines taxes parafiscales et, en particulier, de la cotisation perçue au Budget annexe des prestations familiales agricoles. 
Nous restons volontairement muet sur de multiples autres transformations qui intéressent les bénéfices industriels et commerciaux, les patentes, la taxe sur le chiffre d'affaires.

Nous ne donnons aucun développement de détail sur chacun des nombreux articles qui font l'objet cle ces lois et des décrets d'applications.

Chacun doit posséder un guicle fiscal étoffé pour faire sa déclaration d'impôt, depuis que les citoyens sont pratiquement tous contraints à payer un impôt sur le revenu. Il en est édité de toutes parts et les quotidiens eux-mêmes sont remplis de conseils aux contribuables.

Nous voulons ici donner un aperçu des honnes et matuvaises nouvelles pour le propriétaire forestier.

Au rang des mesures agráables à enregistrer, nous citerons les paragraphes 6,8 et 9 .

L'allègement et la simplification des droits de succession entre époux et parents et enfants sont substantiels et justes en attendant la suppression demandée depuis longtemps.

Nous avons dit, en son temps, ce que nous pensions de la taxe Ramadier qui était un impôt camouflé sur le capital et nous applaudissons à sa mort.

Mais ce qui intéresse au premier chef la forêt privée française est l'exemption des droits de succession et de donation pour. les $3 / 4$ de leur montant, sous les conditions prévues dans la loi de finances du 16 avril 1930.

Cette disposition avait été demandée par notre camarade Robert SÉROT et sans arrêt réclamée depuis 30 ans.

Les héritiers des propriétés forestières feront eux-mêmes le choix entre le sacrifice financicr, assorti de la liberté de ruiner la forêt, et l'avantage fiscal compensé par un engagement de gestion normale.

Nous pensons que presque tous choisiront la deuxième solution et, de ce fait, accepteront la préservation du capital forestier.

C'est un des plus grands actes de politique forestière depuis 30 ans et qui laisse la France sous un des régimes les plus libéraux d'Europe.

Nous devons, par contre, enregistrer avec une certaine inquiétude les majorations révélées aux paragraphes 10 et 11 de notre énumération.

Enfin, il est très difficile de calculer les répercussions; du nouveau système d'impôt sur le revenu des personnes physiques. I1 notıs paraît cependant préférable à l'ancien.

Mais pourquoi chaque avantage, apparemment accordé, est-il immédiatement compensé par un tour de vis sur une autre imposition ou inversement? 
C'est ainsi que si l'échelle des prélèvements par tranches est modifiée en 1959, elle donne naissance à un crédit d'impôt et que la suppression de la taxe proportionnelle nous vaut une taxe complémentaire. Mais ce ne sont pas les mêmes contribuables qui sont touchés par les unes et par les autres mesures.

On a l'impression de dosages savants qui ressemblent à des recettes de cuisine.

Pourquoi avoir gardé ce système invraisemblable de tranches et ce décime supplémentaire, créé le crédit d’impôt, utilisé des pourcentages tels que $9 \%$ ou $19 \%$, Imultiplié les catégories et les imbroglios?

Notre secrétaire d'Etat au Budget est vraiment doué d'un bel humour lorsqu'il répond aux journalistes: "Quoique très simplifiée la formule de déclaration d'impôt a encore besoin de simplification ).

Ne s'arrache-t-on pas les cheveux en lisant au paragraphe 6 de la feuille Annexe $3 \mathrm{~B}$ (un des 13 feuillets à remplir) :

«Reporter dans la colonne 1 le total des chiffres figurant, d'une part, sur la déclaration modèle B (Chapi III, A-7, ligne a) (revenu brut) (cf (3) ci-dessous) et, d'autre part, sur l'annexe $\mathrm{n}^{\circ} 2$ (feuillet jaune), Chap. III, ligne $\mathrm{E}$ et Chap. IV ligne G (cf (3) ci-dessous ) 》.

Doit-on se consoler en pensant que ce n'est pas nouveau et qu'à propos de la taxe civique de 1958 la justification suivante était alors clonnée sur les avertissements:

« La base d'imposition de chaque contribuable est égale à la quote-part du total des valeurs locatives cadastrales des locaux d'habitation de la commune correspondant au loyer matriciel brut en fonction duquel il a été assujetti en 1958 à la contribution mobilière 》?

Serons-nous enclins à demander cles explications aux fonctionnaires spécialisés et à recevoir des réponses telles que celle-ci prêtée à un percepteur d'antan*:

«La contribution des portes et fenêtres est calculée en multipliant le nombre d'ouvertures de chaque catégorie par les tarifs obtenus en modifiant les taux fixés par la loi du 21 avril 1832, article 24, suivant la proportion constatée entre le produit total de la contribution et le chiffre résultant de l'application du tarif légal au nombre des ouvertures de la commune »?

En conclusion et pour finir sur une note optimiste, nous dirons tout le bien que la forêt française doit trouver dans les récentes mesures.

\footnotetext{
Bonne.

*Lalanne (Ph.). - Mort et renouveau de la langue française. Edition
} 
Malgré la complexité du problème, en prenant quelques cas concrets, il nous a semblé que la charge sur le mètre cube de bois produit par le propriétaire serait de $40 \%$ moins élevée dans le cas de refus au bénéfice de la loi Sérot et de $60 \%$ moindre pour la propriété qui en profitera, à partir de 1960.

Puissent les révisions cadastrales en cours ne pas apporter de contreparties qui ruinent ces effets. 